\title{
Quantum regime of a free-electron laser: relativistic approach
}

\author{
Peter Kling ${ }^{1,2}\left(\right.$ Roland Sauerbrey $^{1} \cdot$ Paul Preiss $^{1,2} \cdot$ Enno Giese $^{2,3} \cdot$ \\ Rainer Endrich ${ }^{1,2}$ - Wolfgang P. Schleich ${ }^{2,4}$
}

Received: 16 August 2016 / Accepted: 4 November 2016 / Published online: 15 December 2016

(C) The Author(s) 2016. This article is published with open access at Springerlink.com

\begin{abstract}
In the quantum regime of the free-electron laser, the dynamics of the electrons is not governed by continuous trajectories but by discrete jumps in momentum. In this article, we rederive the two crucial conditions to enter this quantum regime: (1) a large quantum mechanical recoil of the electron caused by the scattering with the laser and the wiggler field and (2) a small energy spread of the electron beam. In contrast to our recent approach based on nonrelativistic quantum mechanics in a co-moving frame of
\end{abstract}

This article is part of the topical collection "Enlightening the World with the Laser" - Honoring T. W. Hänsch guest edited by Tilman Esslinger, Nathalie Picqué, and Thomas Udem.

It is a great honor and pleasure for us to dedicate this article to Theodor W. Hänsch on the occasion of his 75 th birthday. On first sight, there seems to be no connection between his research interests and the field of free-electron lasers (FELs). However, a closer examination reveals once more his crucial influence in shaping a new field. Indeed, in one [1] of the pioneering articles on FEL theory, W. B. Colson acknowledges 'fruitful discussions,' he had with Hänsch on this topic. Therefore, we find it appropriate to contribute an article devoted to the Quantum FEL to this Special Issue celebrating the scientific achievements of Theodor W. Hänsch. Our warmest wishes, and Happy Birthday Ted.

Peter Kling

peter.kling@uni-ulm.de

1 Helmholtz-Zentrum Dresden-Rossendorf eV, 01328 Dresden, Germany

2 Institut für Quantenphysik and Center for Integrated Quantum Science and Technology (IQST), Universität Ulm, Albert-Einstein-Allee 11, 89081 Ulm, Germany

3 Department of Physics, University of Ottawa, 25 Templeton Street, Ottawa, ON K1N 6N5, Canada reference, we now pursue a model in the laboratory frame employing relativistic quantum electrodynamics.

\section{Introduction}

An important insight gained already in the early days of the free-electron laser (FEL), especially in the articles by Colson [1] and Hopf et al. [2], was that the FEL can be completely understood in terms of classical physics [3]. However, recent years have seen a rising interest in a novel regime of FEL operation where quantum effects become crucial $[4,5]:$ the so-called quantum regime or Quantum FEL. For example, in Refs. [4, 5] the authors consider the Heisenberg equations of motion while in Ref. [6] as well as in Refs. [7, 8] formalisms in second quantization are discussed. Moreover, a quantum fluid model is developed in Ref. [9] and in Refs. [10, 11] the authors use the KleinGordon equation coupled to classical electromagnetic fields to derive a dispersion relation.

While in all these approaches the respective sets of equations were solved and the quantum regime was identified as one limit of the solution, our article [12] on the Quantum FEL takes a different route: We search in the dynamical

4 Texas A\&M University Institute for Advanced Study (TIAS), Institute for Quantum Science and Engineering (IQSE), Department of Physics and Astronomy, Texas A\&M University, College Station, TX 77843-4242, USA 
equations for a regime where quantum mechanics becomes prevalent and solve these equations in an asymptotic way. To achieve this goal, we have used a nonrelativistic approach in the co-moving Bambini-Renieri frame [13, 14]. In contrast, we develop in the present article a fully relativistic model in the laboratory frame and derive within this framework the conditions to enter the quantum regime.

\subsection{Conditions for Quantum FEL}

In Refs. [12, 15-17], we have shown that quantum effects become important for the FEL dynamics when the discrete quantum mechanical recoil due to the scattering of the electron is the dominating momentum scale. Moreover, we have defined the quantum regime of the FEL as the limit where the electron occupies only two resonant momentum states giving rise to a system analogous to the Jaynes-Cummings model [18], where a two-level atom interacts with a quantized mode of the radiation field.

This transition to the quantum regime is quantified by two conditions: The first one relates to the 'quantum parameter' $\alpha$ which is defined as the ratio of the coupling strength between the electron and the fields, and a frequency connected to the quantum mechanical recoil. To be in the quantum regime, $\alpha$ has to be much smaller than unity.

The second requirement for the Quantum FEL demands the width $\Delta p$ of the initial momentum distribution to be smaller, or of the order of the recoil.

To analyze these two conditions for a possible experimental realization, we have expressed $\alpha$ and $\Delta p$ in the laboratory frame by transforming from the co-moving frame to the laboratory frame. With this procedure, we have obtained [12] the requirement ${ }^{1}$

$\alpha \equiv \frac{\gamma^{2} g \sqrt{n}}{\hbar\left(k_{\mathrm{L}}+k_{\mathrm{W}}\right)^{2} /(2 m)} \ll 1$

for the quantum parameter $\alpha$ where $g, n, m$ and $\hbar$ denote the coupling constant between the electron and the fields, the number of laser photons, the mass of the electron and the reduced Planck constant, respectively, while $\gamma$ is the scaled energy of the electron. The wave numbers of the laser field and the wiggler are given by $k_{\mathrm{L}}$ and $k_{\mathrm{W}}$, respectively.

On the other hand, we have found the condition [12]

$\frac{\Delta \gamma}{\gamma}<4 \gamma \frac{\lambda_{\mathrm{C}}}{\lambda_{\mathrm{W}}}$

${ }^{1}$ In Ref. [12], we have rewritten $\alpha$ in terms of the wiggler parameter $a_{0}$, the electron density $n_{\mathrm{e}}$, the classical electron radius $r_{\mathrm{e}}$ and the Compton wavelength $\lambda_{\mathrm{C}}$. Indeed, the form

$\alpha=\frac{1}{\gamma^{3}} \frac{a_{0} \sqrt{r_{\mathrm{e}} n_{\mathrm{e}}}}{32 \sqrt{\pi}} \frac{\lambda_{\mathrm{W}}^{5 / 2}}{\lambda_{\mathrm{C}}^{3 / 2}}$

is more convenient to compare to experimental parameters. on the relative energy spread $\Delta \gamma / \gamma$ of the electron beam with the Compton wavelength $\lambda_{\mathrm{C}}$ of an electron and the wiggler wavelength $\lambda_{\mathrm{W}} \equiv 2 \pi / k_{\mathrm{W}}$.

However, we could have taken a more direct route avoiding the detour to the Bambini-Renieri frame and perform the calculations in the laboratory frame. The price for this procedure is of course that we have to use the more tedious methods of relativistic quantum electrodynamics instead of the straightforward nonrelativistic computations.

Nonetheless, the physical situation in both frames of reference is the same and we should find analogous results. Hence, we formulate the goal of the present article: Find the conditions for the Quantum FEL in a fully relativistic approach, and compare them to the ones obtained in the framework of the Bambini-Renieri frame.

\subsection{Outline}

Our article is organized as follows: In Sect. 2, we review a model $[19,20]$ for a quantum theory of the FEL in the laboratory frame based on the Furry or bound interaction picture [21] as well as on the Volkov solution [22].

With the help of this model, we derive in Sect. 3 the conditions for the Quantum FEL in the laboratory frame, which turn out to be equivalent to the ones of Ref. [12]. For our calculations, we restrict ourselves to a single-electron model which limits us to the low-gain regime of FEL operation. Moreover, we solve the FEL dynamics in a perturbative manner, which is only valid for short times. Despite these simplifications, we are able to identify the important parameters governing the transition from the classical to the quantum regime of the FEL. In Sect. 4, we summarize our results.

Appendices A and B give a more detailed description of the Furry picture and the Volkov solution, respectively. In Appendix C, we present the calculations of second-order perturbation theory to prove also in the laboratory frame that multi-photon processes are suppressed in the Quantum FEL.

\section{Quantum model of FEL in laboratory frame}

Due to the relativistic velocities of the electrons in the laboratory frame, we have to use methods of relativistic quantum electrodynamics to establish a quantum theory of the FEL in this frame of reference. A suitable model, introduced in Refs. [19, 20], can be found in the so-called Furry or bound interaction picture [21]. In this description, the effects of the wiggler field, which is modeled as a classical and fixed quantity, are incorporated into the free dynamics of the electron while the quantized laser field acts as a perturbation of this motion. 
Due to difficulties in treating multi-photon processes [23], which are crucial for the classical FEL [24], this relativistic model was discarded and theories were developed [25] in the co-moving Bambini-Renieri frame. ${ }^{2}$ However, since processes involving more than one photon are strongly suppressed [12] in the Quantum FEL, we assume that the Furry picture is a good starting point for our investigations.

To introduce our model, we first consider the electromagnetic fields and then turn to the electron. We conclude by discussing the interaction between the electron and the fields, and deriving the Hamiltonian for the FEL in the laboratory frame.

\subsection{Wiggler and laser fields}

Due to its high intensity, we assume that the wiggler field can be treated as a classical and fixed electromagnetic field. In contrast, we describe the laser field in second quantization.

We model the four-potentials

$\hat{A}_{\mathrm{L}}^{\mu}(x) \equiv \mathcal{A}_{\mathrm{L}}\left(\hat{a}_{\mathrm{L}} \epsilon^{\mu} \mathrm{e}^{-i k_{\mathrm{L}} \cdot x}+\right.$ h.c. $)$

and

$A_{\mathrm{W}}^{\mu}(x) \equiv \tilde{\mathcal{A}}_{\mathrm{W}}\left(\epsilon^{\mu} \mathrm{e}^{-i k_{\mathrm{W}} \cdot x}+\right.$ c.c. $)$

of the laser field and of the wiggler as two counterpropagating one-mode plane waves with the vacuum amplitude $\mathcal{A}_{\mathrm{L}}$ for the laser, and the amplitude $\tilde{\mathcal{A}}_{\mathrm{W}}$ for the wiggler. The four-vector $x \equiv x^{\mu} \equiv(c t, \boldsymbol{r})$ specifies time $t$ and position $\boldsymbol{r}$ in the four-dimensional Minkowski space, while $c$ denotes the speed of light.

The photon annihilation and creation operators $\hat{a}_{\mathrm{L}}$ and $\hat{a}_{\mathrm{L}}^{\dagger}$ fulfill the commutation relation $\left[\hat{a}_{\mathrm{L}}, \hat{a}_{\mathrm{L}}^{\dagger}\right]=1$, while the potentials obey circular polarization with the polarization vectors $\epsilon \cdot \epsilon=\epsilon^{*} \cdot \epsilon^{*}=0$ and $\epsilon \cdot \epsilon^{*}=-1$, written in a four-dimensional form.

The scalar products of the four-wave vectors $k_{\mathrm{L}}$ and $k_{\mathrm{W}}$ with themselves vanish for plane waves, i.e., $k_{\mathrm{L}} \cdot k_{\mathrm{L}}=k_{\mathrm{W}} \cdot k_{\mathrm{W}}=0$, provided we consider the case of a laser wiggler. In the case of a magnetostatic wiggler, $k_{\mathrm{W}} \cdot k_{\mathrm{W}}$ would give a nonzero contribution, because the zeroth component which corresponds to the frequency of the field is zero in this case.

\footnotetext{
${ }^{2}$ Even the relativistic quantum theory of the FEL pursued in Refs. [24, 26] is effectively in the Bambini-Renieri frame. Indeed, this approach begins by using the Klein-Gordon equation in the laboratory frame, but after several transformations of variables one reproduces the same equations of motion as if one had considered the comoving frame from the start.
}

Since for an experimental operation of a Quantum FEL a laser wiggler or 'optical undulator' [27-30] was proposed [31], we concentrate in this article on this kind of undulator. However, even for a magnetostatic wiggler we find results [20] analogous to the optical ones, because in its rest frame the electron experiences the wiggler as a counterpropagating wave at approximately the speed of light $[13,14]$.

\subsection{Electron in wiggler field}

To simplify our calculations, we use the Klein-Gordon instead of the Dirac equation, which is justified since spin effects in the FEL can be neglected [32]. Indeed, according to Ref. [23] the corrections due to the spin scale with the ratio of the energy of a single laser photon and the kinetic energy of the electron in the laboratory frame. Although one expects that in the quantum regime a genuine quantum mechanical quantity such as the photon energy plays a significant role, it is still much smaller than the energy of the massive electron moving at relativistic velocities. Hence, we can safely neglect spin effects in any description of the FEL-in the classical as well as in the quantum regime.

The unperturbed dynamics of the Klein-Gordon field operator $\hat{\varphi}(x)$ in the Furry picture is determined by the Klein-Gordon equation including the interaction of the electron with the wiggler field, which is derived in Appendix A. To obtain a particle interpretation of the electron field, we first have to find a solution of the corresponding Klein-Gordon equation

$\left[\left(i \hbar \partial^{\mu}-\mathrm{e} A_{\mathrm{W}}^{\mu}(x)\right)^{2}-m_{0}^{2} c^{2}\right] \varphi(x)=0$

for the 'classical' field $\varphi(x)$. Here, $m_{0}$ and $e$ describe the mass of the electron and the elementary charge, respectively.

The Volkov solution of Eq. (5) is derived in Appendix B and reads

$\varphi_{p}(x) \equiv \frac{1}{\sqrt{2 \gamma m c V}} \mathrm{e}^{-i p \cdot x / \hbar}$

where

$p^{\mu} \equiv p_{\mathrm{f}}^{\mu}+\frac{\mathrm{e}^{2} \tilde{\mathcal{A}}_{\mathrm{W}}^{2}}{p_{\mathrm{f}} \cdot k_{\mathrm{W}}} k_{\mathrm{W}}^{\mu}$,

denotes the effective four-momentum of the electron in the wiggler and $p_{\mathrm{f}}$ is the momentum of the free electron before it enters the wiggler.

The effective mass $m$ is connected to $p$ and to the 'free mass' $m_{0}$ via the relation

$p \cdot p=m_{0}^{2} c^{2}+2 \mathrm{e}^{2} \tilde{\mathcal{A}}_{\mathrm{W}}^{2} \equiv m^{2} c^{2}$. 
The fact that we are dealing with the effective electron momentum, $p$, instead of the free one, $p_{\mathrm{f}}$, can be interpreted by a dressed electron model, where the effects of the external field $A_{\mathrm{W}}$ are already taken into account in form of the effective mass and momentum.

Other quantities that occur in the Volkov solution, Eq. (6), are the relativistic factor $\gamma \equiv p_{0} / m c$ and the quantization volume $V$.

In order to arrive at the familiar particle interpretation of the field operator $\hat{\varphi}(x)$, we expand [20] it in terms of the Volkov solutions, Eq. (6), and arrive at

$\hat{\varphi}(x)=\sum_{p} \varphi_{p}(x) \hat{b}_{p}$

where $\hat{b}_{p}$ annihilates an electron with momentum $p$ and $\hat{b}_{p}^{\dagger}$ creates one.

One could argue that the creation and annihilation operators for a scalar field fulfill bosonic commutation relations, $\left[\hat{b}_{p}, \hat{b}_{p^{\prime}}^{\dagger}\right]=\delta_{p, p^{\prime}}$, instead of the required anti-commutator relations for the fermionic electrons. However, since we are describing a single-electron model, the difference in the occupations of fermionic and bosonic many-particle quantum states does not enter into our theory.

We also emphasize that the expansion, Eq. (9), considers only particle operators and the corresponding positive energy solutions. Indeed, we have discarded anti-particle operators with negative energy solutions in complete analogy to Ref. [20].

\subsection{Electron in wiggler plus laser field}

The Hamiltonian

$\hat{H}(t) \equiv \mathrm{e} \int \mathrm{d}^{3} r \hat{j}(x) \cdot \hat{A}_{\mathrm{L}}(x)$

describing the interaction between the electron and the electromagnetic fields in the Furry picture is derived in Appendix A and couples the electron current $\hat{j}^{\mu}$ to the fourpotential of the quantized laser field $\hat{A}_{\mathrm{L}}^{\mu}$.

The components

$$
\begin{aligned}
\hat{j}^{\mu}(x) \equiv & c\left[\hat{\varphi}^{\dagger}(x) i \hbar \partial^{\mu} \hat{\varphi}(x)-\left(i \hbar \partial^{\mu} \hat{\varphi}^{\dagger}(x)\right) \hat{\varphi}(x)\right] \\
& +2 \mathrm{e} A_{\mathrm{W}}^{\mu}(x) \hat{\varphi}^{\dagger}(x) \hat{\varphi}(x)
\end{aligned}
$$

of the current corresponding to the electron in the wiggler field involve the field operator $\hat{\varphi}$ of the Klein-Gordon field and the four-potential $A_{\mathrm{W}}$ of the classical wiggler field.

We assume that the electron is injected parallel to the wiggler axis which we denote as the $z$-direction. Hence, all transverse components of the initial momentum vanish. Since the laser field and the wiggler field form a collinear geometry their phases also depend only on $z$ and due to the interaction with these fields, the electron dynamics is maintained in the $z$-direction. Hence, we can consider an effective one-dimensional theory for the FEL dynamics.

Thus, by examining the Volkov solution, Eq. (6), and the electron current, Eq. (11), we observe that only the transverse components of the current, proportional to $A_{\mathrm{W}}^{\mu}(x)$, couple to the laser field $\hat{A}_{\mathrm{L}}^{\mu}(x)$, because in our one-dimensional model we have

$p \cdot \hat{A}_{\mathrm{L}}=p_{\mathrm{f}} \cdot \hat{A}_{\mathrm{L}}=0$.

For our choice of circular polarization, we can derive from Eqs. (3), (4), (9), (10) and (11) the Hamiltonian

$\hat{H}(t)=\int_{V} \mathrm{~d}^{3} r\left[\hat{\mathcal{H}}_{\mathrm{em}}(x)+\hat{\mathcal{H}}_{\mathrm{abs}}(x)\right]$

where

$\hat{\mathcal{H}}_{\mathrm{em}}(x) \equiv \frac{\hbar g}{V} \sum_{p^{\prime}, p^{\prime \prime}} \frac{\mathrm{e}^{i \Phi(x)}}{\sqrt{\gamma^{\prime} \gamma^{\prime \prime}}} \hat{b}_{p^{\prime}}^{\dagger} \hat{b}_{p^{\prime \prime}} \hat{a}_{\mathrm{L}}^{\dagger}$

describes the emission of photons into the laser field with the phase

$\Phi(x) \equiv \frac{1}{\hbar}\left[p^{\prime \mu}-p^{\prime \prime \mu}+\hbar\left(k_{\mathrm{L}}^{\mu}-k_{\mathrm{W}}^{\mu}\right)\right] x_{\mu}$

while

$\mathcal{H}_{\mathrm{abs}}(x)=\left(\mathcal{H}_{\mathrm{em}}(x)\right)^{\dagger}$

is the corresponding absorption term.

The coupling constant $g$ is defined as

$g \equiv \frac{\mathrm{e}^{2} \mathcal{A}_{\mathrm{L}} \tilde{\mathcal{A}}_{\mathrm{W}}}{\hbar m}$

in complete analogy to Ref. [12].

The dynamics of the system consisting of electron and laser field is given by the state vector $|\Psi(t)\rangle$. In the following, we write $|\Psi(t)\rangle$ in terms of photon number states $|n\rangle$ of the laser field and momentum eigenstates $|p\rangle \equiv\left|1_{p}\right\rangle=\hat{b}_{p}^{\dagger}|0\rangle$ of the electron. The vacuum $|0\rangle$ of the electron field is defined by the relation $\hat{b}_{p}|0\rangle=0$, valid for all momenta $p$. Projecting $\langle n, p|$ on the total state $|\Psi(t)\rangle$ and taking the modulus square, that is, $|\langle n, p \mid \Psi(t)\rangle|^{2}$, gives us the joint probability to find $n$ photons in the laser mode and the momentum $p$ of the electron.

\section{Quantum FEL in the laboratory frame}

In this section, we analyze the FEL dynamics in the laboratory frame governed by the Hamiltonian, Eq. (13), and ultimately derive the necessary requirements to enter the quantum regime. First, we investigate the microscopic processes for the FEL from the point of view of energy-momentum conservation; then, we use first-order perturbation theory 
to solve the equations of motion for the system. Finally, we identify the Quantum FEL as the limit where single-photon processes dominate, while multi-photon transitions are suppressed.

\subsection{Resonance condition}

For our study of the basic processes in the FEL dynamics, it is convenient to consider energy-momentum conservation in the formulation of co- and contravariant four-vectors. As described in the previous section, we use the effective four-momentum

$p^{\mu} \equiv\left(p_{0}, 0,0, p_{z}\right) \equiv \gamma m c(1,0,0, \beta)$

of the electron in the wiggler instead of the free momentum

$p_{\mathrm{f}}^{\mu} \equiv \gamma_{0} m_{0} c\left(1,0,0, \beta_{0}\right)$,

where we have chosen the direction of the propagation parallel to the $z$-axis. Moreover, we have defined the relativistic factors

$\gamma \equiv \frac{1}{\sqrt{1-\beta^{2}}}$

and

$\gamma_{0} \equiv \frac{1}{\sqrt{1-\beta_{0}^{2}}}$

together with the scaled velocities $\beta$ and $\beta_{0}$ such that the four-momentum fulfills the conditions $p \cdot p=m^{2} c^{2}$ and $p_{\mathrm{f}} \cdot p_{\mathrm{f}}=m_{0}^{2} c^{2}$.

The reason for considering the effective momentum $p$ is its appearance in the Volkov solution, Eq. (6), and in the phase, Eq. (15), of the Hamiltonian, Eq (13). Classically it can be understood by identifying $p^{\mu}$ as an effective longitudinal momentum which contains the effects of the transverse motion of the electron in the wiggler due to the Lorentz force [33].

The microscopic processes of the FEL can be described in terms of Compton scattering, where $l$ wiggler photons with the four-wave vector

$k_{\mathrm{W}}^{\mu}=k_{\mathrm{W}}(1,0,0,-1)$

and $l$ laser photons with the four-wave vector

$k_{\mathrm{L}}^{\mu}=k_{\mathrm{L}}(1,0,0,1)$

interact with an electron, which possesses the initial momentum $p^{\mu}$.

Due to energy-momentum conservation, the final momentum $p^{\prime \mu}$ of the electron reads [34]

$p^{\prime \mu}=p^{\mu} \mp l \hbar\left(k_{\mathrm{L}}^{\mu}-k_{\mathrm{W}}^{\mu}\right)$, where the minus sign occurs in the case of emission of $l$ laser photons and absorption of $l$ wiggler photons ('emission'), while the plus sign describes the opposite process ('absorption').

We use the relation, Eq. (8), in order to eliminate the final momentum $p^{\prime \mu}$ in Eq. (24). After straightforward algebra, we observe, for fixed values of the wave numbers $k_{\mathrm{L}}$ and $k_{\mathrm{W}}$, the resonance condition

$\beta=\frac{k_{\mathrm{L}}-k_{\mathrm{W}}}{k_{\mathrm{L}}+k_{\mathrm{W}}} \pm l \frac{2 k_{\mathrm{W}}}{k_{\mathrm{L}}+k_{\mathrm{W}}} \frac{\hbar k_{\mathrm{L}}}{\gamma m c}$.

For the time being, we neglect the recoil term in Eq. (25), since the photon momentum $\hbar k_{\mathrm{L}}$ is much smaller than the electron momentum $\gamma m c$, as well as $k_{\mathrm{W}} \ll k_{\mathrm{L}}$. Indeed, we would need an unrealistic high number $l$ of scattered photons to obtain noticeable corrections to the leading term.

Hence, we obtain for classical resonance

$\beta \cong \frac{k_{\mathrm{L}}-k_{\mathrm{W}}}{k_{\mathrm{L}}+k_{\mathrm{W}}} \equiv \beta_{\mathrm{BR}}$

where only the wave numbers of the fields occur and no term with $\hbar$ is present. As shown in Appendix A of Ref. [12], Eq. (26) gives the velocity $\beta_{\mathrm{BR}}$ of the BambiniRenieri frame relative to the laboratory frame.

Alternatively we can derive the resonance condition for $k_{\mathrm{L}}$ with fixed $k_{\mathrm{W}}$ and $\gamma$. An analogous calculation [26] yields the expression ${ }^{3}$

$k_{\mathrm{L}} \cong \frac{4 \gamma_{0}^{2} k_{\mathrm{W}}}{\left(1+a_{0}^{2}\right)}$,

where we have defined [35] the wiggler parameter $a_{0} \equiv \mathrm{e} \sqrt{2} \tilde{\mathcal{A}}_{\mathrm{W}} /\left(m_{0} c\right)$. Moreover, we have made the approximations $1+\beta \cong 2$ and $\gamma m \cong \gamma_{0} m_{0}$, which can be easily derived from $\beta \lesssim 1$ and $p_{\mathrm{f}} \cdot k_{\mathrm{W}}=p \cdot k_{\mathrm{W}}$.

We emphasize that neglecting the recoil contributions in Eq. (25) is a reasonable procedure to estimate the resonant velocity for the electron relative to the laboratory frame. However, the recoil is the reason for gain in the FEL regardless of whether we consider the classical regime, where many photons are scattered, but the discrete nature of the jumps is washed out, or we are in the quantum regime where only few photons are scattered. Hence, we have to consider the relative velocity of the electron with respect to the resonant one, Eq. (26), in a complete theory of the FEL.

In other words, the recoil term in Eq. (25) gives a nonrelativistic correction to Eq. (26) which may or may not be neglected, depending on the observable of interest. The

\footnotetext{
3 We note that the result for the resonant wave number $k_{\mathrm{L}}$ differs by a factor of 2 from the one for a magnetostatic wiggler [26].
} 
recoil corrections in Eq. (25) are much smaller than unity while the scaled velocity $\beta_{\mathrm{BR}}$ of the Bambini-Renieri frame is close to unity. For this reason we can identify the recoil terms as nonrelativistic which justifies the approach in the co-moving frame of Ref. [12].

We now further rewrite the expression in Eq. (25). With the help of the identity

$\gamma_{\mathrm{BR}}=\frac{k_{\mathrm{L}}+k_{\mathrm{W}}}{2 \sqrt{k_{\mathrm{L}} k_{\mathrm{W}}}}$

derived in Appendix A of Ref. [12], we obtain the form

$\frac{2 k_{\mathrm{W}}}{k_{\mathrm{L}}+k_{\mathrm{W}}} \frac{\hbar k_{\mathrm{L}}}{\gamma m c}=\frac{q}{2 \gamma_{\mathrm{BR}}^{2} \gamma m c}$

for the recoil term in Eq. (25). Here we have defined the quantum mechanical recoil

$q \equiv \hbar\left(k_{\mathrm{L}}+k_{\mathrm{W}}\right)$

which is the change of the electron momentum in $z$-direction due to a single scattering process according to Eq. (24).

Finally we cast Eq. (25) into the form

$\beta=\beta_{\mathrm{BR}} \pm l \frac{\mathcal{Q}}{2}$

where we have recalled the definition of $\beta_{\mathrm{BR}}$, Eq. (26), and have introduced the dimensionless recoil parameter

$\mathcal{Q} \equiv \frac{\hbar\left(k_{\mathrm{L}}+k_{\mathrm{W}}\right)}{\gamma^{3} m c}$.

In the transition from Eq. (29) to (32), we have additionally approximated $\gamma_{\mathrm{BR}} \cong \gamma$ since all processes take place close to the classical resonance.

\subsection{Dynamics}

The dynamics of the state $|\Psi(t)\rangle$ describing the motion of the electron and the laser field during an interaction time $t$ is given by

$|\Psi(t)\rangle=\hat{\mathcal{U}}(t)|\Psi(0)\rangle$

with the time evolution operator [36]

$$
\begin{aligned}
\hat{\mathcal{U}}(t) \equiv & \sum_{j=0}^{\infty}\left(\frac{-i}{\hbar}\right)^{j} \int_{0}^{t} \mathrm{~d} t_{j} \int_{0}^{t_{j}} \mathrm{~d} t_{j-1} \cdots \int_{0}^{t_{2}} \mathrm{~d} t_{1} \\
& \times \hat{H}\left(t_{j}\right) \hat{H}\left(t_{j-1}\right) \cdots \hat{H}\left(t_{2}\right) \hat{H}\left(t_{1}\right)
\end{aligned}
$$

originating from the time-dependent Hamiltonian $\hat{H}(t)$ given by Eq. (13).

Following Ref. [19], we extend the spatial integration in the $z$-direction to infinity in Eq. (13), while considering a finite integration time $t$. This way, the integral over space leads to a delta function for the momentum.
In analogy to Ref. [12], we use perturbation theory to solve the dynamics, i.e., we subsequently evaluate the individual terms of the sum in Eq. (34). However, before we proceed, we examine the action of the Hamiltonian, Eq. (13), on a state.

As mentioned above, the Hamiltonian density can be split into an emission $\hat{\mathcal{H}}_{\text {em }}$ and absorption term $\hat{\mathcal{H}}_{\text {abs. }}$ The combination of the creation and annihilation operators of the electron and of the laser field ensures that in the time evolution Eq. (33), for the initial state $\left|n, p_{z}\right\rangle$ consisting of a photon number state $|n\rangle$ and a momentum eigenstate $\left|p_{z}\right\rangle$, only a state

$|\Psi(t)\rangle=\sum_{l=-n}^{\infty} c_{l}(t)\left|n+l, p_{z}-l q\right\rangle$

can be generated, where $l$ photons are emitted into, or absorbed from the laser field while the electron looses or gains the momentum $l q$. The quantum mechanical recoil $q \equiv \hbar\left(k_{\mathrm{L}}+k_{\mathrm{W}}\right)$ is defined according to Eq. (30).

This effect corresponds to momentum conservation in Eq. (24) but not to energy conservation. Due to the finite integration time, we will see that the zeroth component of the four-momentum, i.e., the energy, is not exactly conserved leading to combinations of sinc functions in the probabilities.

The expansion coefficients $c_{l}$ in Eq. (35) represent probability amplitudes for a process involving $l$ photons and the electron recoiling $l$ times. The basis states $\left|n \pm l, p_{z} \mp l q\right\rangle$ used in this expansion are also known [37] as 'scattering basis.'

In contrast to Eq. (35), we allow in the following a distribution of the initial momenta with a nonzero width. In accordance with Ref. [12], we assume the initial condition

$c_{l}\left(p_{z} ; 0\right)=\delta_{l, 0} \psi\left(p_{z}\right)$

which describes the situation where the initial state of the system is characterized by a photon number state with $n$ photons in the laser field and by the wave function $\psi=\psi\left(p_{z}\right)$ of the electron depending on the momentum $p_{z}$ in $z$-direction.

\subsection{Single-photon processes}

Finally, we are in the position to calculate the probability densities $\left|c_{1}\right|^{2}$ and $\left|c_{-1}\right|^{2}$ of single-photon emission and absorption, respectively, in lowest order of perturbation theory. The resulting expressions for $\left|c_{ \pm 1}\right|^{2}$ involve the selectivity functions $\mathcal{S}_{ \pm}$which contain the resonance conditions for absorption and emission discussed in Sect. 3.1. Moreover, they are also sensitive to the initial momentum distribution of the electron. Indeed, the displacement of $\mathcal{S}_{+}$relative to $\mathcal{S}_{-}$, and its size compared to the width of 
the momentum distribution leads to the conditions for the Quantum FEL.

\subsubsection{Selectivity functions}

We start our analysis by computing the first terms of Eq. (34), which yields

$$
\begin{aligned}
& {\left[1-\frac{i}{\hbar} \int_{0}^{t} \mathrm{~d} t_{1} \int \mathrm{d}^{3} r\left(\hat{\mathcal{H}}_{\mathrm{em}}\left(t_{1}, \boldsymbol{r}\right)+\hat{\mathcal{H}}_{\mathrm{abs}}\left(t_{1}, \boldsymbol{r}\right)\right)\right]\left|n, p_{z}\right\rangle} \\
& =\left|n, p_{z}\right\rangle+c_{1}\left|n+1, p_{z}-q\right\rangle+c_{-1}\left|n-1, p_{z}+q\right\rangle,
\end{aligned}
$$

when applied to the state $\left|n, p_{z}\right\rangle$. As expected, this state has the same form as the expansion Eq. (35), with only three nonvanishing contributions, that is, for $l=0$ as well as for $l=1$ and $l=-1$.

After straightforward algebra and using the initial condition Eq. (36), we arrive at the probability densities

$\left|c_{1}\right|^{2}=\left(\frac{g t}{\gamma}\right)^{2}(n+1) \mathcal{S}_{+}^{2}\left|\psi\left(p_{z}\right)\right|^{2}$

for single-photon emission, and

$\left|c_{-1}\right|^{2}=\left(\frac{g t}{\gamma}\right)^{2} n \mathcal{S}_{-}^{2}\left|\psi\left(p_{z}\right)\right|^{2}$

for single-photon absorption. Here we have weighted every momentum eigenstate with the corresponding initial momentum distribution $\left|\psi\left(p_{z}\right)\right|^{2}$.

Moreover, we have neglected the small energy change in the pre-factor yielding $\sqrt{\gamma^{\prime} \gamma} \cong \gamma$ and have introduced the selectivity functions

$\mathcal{S}_{ \pm} \equiv \operatorname{sinc}\left[\frac{c t}{2 \hbar}\left(p_{0}\left(p_{z} \mp q\right)-p_{0} \pm \hbar\left(k_{\mathrm{L}}-k_{\mathrm{W}}\right)\right)\right]$

which characterize the position and the width of the resonant energies.

To simplify the argument of $\mathcal{S}_{ \pm}$, Eq. (40), we expand [19] the final energy

$p_{0}\left(p_{z} \mp q\right)=\sqrt{\left(p_{z} \mp q\right)^{2}+m^{2} c^{2}}$

to second order in the recoil, that is,

$p_{0}\left(p_{z} \mp q\right) \cong p_{0} \mp \frac{p_{z} q}{p_{0}}+\frac{q^{2}}{2 p_{0}}\left(1-\frac{p_{z}^{2}}{p_{0}^{2}}\right)$,

while keeping in mind that $q$ is always a small quantity in comparison with the electron momentum, characterized by $\gamma \gg 1$. Moreover, we have used the fact that $p_{\mu}^{\prime}$ fulfills the relation $p^{\prime} \cdot p^{\prime}=m^{2} c^{2}$ due to Eq. (8).
We finally obtain

$\mathcal{S}_{ \pm}=\operatorname{sinc}\left[\frac{1}{2}\left(k_{\mathrm{L}}+k_{\mathrm{W}}\right) c t\left(\beta-\beta_{\mathrm{BR}} \mp \frac{\mathcal{Q}}{2}\right)\right]$

where we have recalled the definitions, Eqs. (26) and (32), of the scaled velocity $\beta_{\mathrm{BR}}$ of the Bambini-Renieri frame and of the recoil parameter $\mathcal{Q}$, respectively.

Hence, the resonances are centered around $\beta_{\mathrm{BR}}$ and shifted to the left for absorption or to the right for emission, by the recoil term $\mathcal{Q} / 2$. This structure is already predicted by energy-momentum conservation ${ }^{4}$ in Eq. (31).

\subsubsection{Conditions}

We now use the results of first-order perturbation theory to derive the requirements for realizing the Quantum FEL. Here we follow closely the arguments of Ref. [12].

Quantum parameter Therefore, we introduce the recoil frequency

$\omega_{\mathrm{r}} \equiv \frac{c}{2}\left(k_{\mathrm{L}}+k_{\mathrm{W}}\right) \frac{\mathcal{Q}}{2}=\frac{\hbar\left(k_{\mathrm{L}}+k_{\mathrm{W}}\right)^{2}}{4 \gamma^{3} m}$

in the laboratory frame. The connection to the corresponding quantity, introduced in Eq. (6) of Ref. [12] in the Bambini-Renieri frame, is achieved by considering the product $\omega_{\mathrm{r}} t$ and not by just transforming $\omega_{\mathrm{r}}$. The latter transformation yields a factor of $\gamma^{-2}$. On the other hand, the interaction time $t^{\prime}$ in the Bambini-Renieri frame has to be translated via $t^{\prime} \rightarrow t / \gamma$ to $t$ in the laboratory frame, due to time dilatation. These two steps provide us with the dependency on $\gamma^{-3}$ of $\omega_{\mathrm{r}} t$ in the laboratory frame as predicted in Eq. (44).

As depicted in Fig. 1, the parameter $\omega_{\mathrm{r}} t$ is a measure for the width and the separation of the resonances. In the classical case, that is, for $\omega_{\mathrm{r}} t \ll 1$, the selectivity functions overlap. For large recoil, that is, for $\omega_{\mathrm{r}} t \gg 1$, these functions are well separated and possess a small width. This limit with two distinguishable and sharp resonances, defines the quantum regime of the FEL.

For the asymptotic expansion of the time evolution, Eq. (34), to converge, we require that the expansion parameter $g t \sqrt{n} / \gamma$, which emerges in Eqs. (38) and (39) is small, i.e., $g t \sqrt{n} / \gamma \ll 1$. We emphasize that the connection to the parameter $g t^{\prime} \sqrt{n}$ of the nonrelativistic theory of Ref. [12] is again established by time dilatation $t^{\prime} \rightarrow t / \gamma$.

\footnotetext{
${ }^{4}$ In contrast to the derivation of $\mathcal{Q}$ in Eq. (31), we did not have to make the approximation $\gamma_{\mathrm{BR}} \approx \gamma$ when we derived Eq. (43). This difference stems from the fact that we have performed a Taylor expansion of the relativistic square root in Eq. (41) to linearize the argument of the selectivity functions.
} 

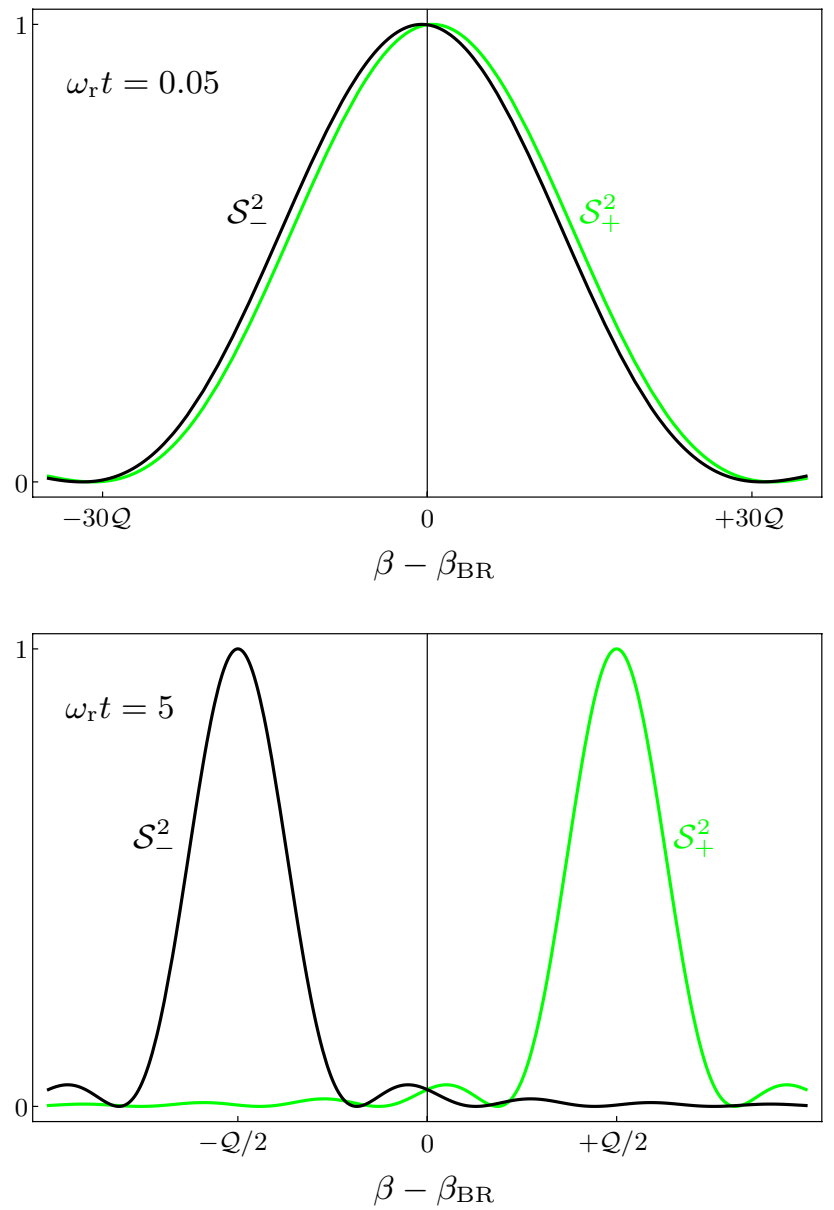

Fig. 1 Transition from the classical FEL (top) to the Quantum FEL (bottom) illustrated by the behavior of the selectivity functions $\mathcal{S}_{ \pm}^{2}$, Eq. (43), for single-photon emission $\mathcal{S}_{+}^{2}$ (green curve) and absorption $\mathcal{S}_{-}^{2}$ (black curve) as functions of the scaled velocity $\beta$ of the electron in the laboratory frame. While $\mathcal{S}_{+}^{2}$ and $\mathcal{S}_{-}^{2}$ overlap in the classical regime shown here for $\omega_{\mathrm{r}} t=0.05$, we obtain well-separated resonances for the Quantum FEL exemplified by $\omega_{\mathrm{r}} t=5$

The ratio $\alpha$ of the expansion parameter $g t \sqrt{n} / \gamma$ to $\omega_{\mathrm{r}} t$ can be written as

$\alpha \equiv \gamma^{2} \frac{g \sqrt{n}}{q^{2} /(2 m \hbar)} \ll 1$

where the inequality emerges for the quantum case $\omega_{\mathrm{r}} t \gg 1$. That is why we call $\alpha$ the 'quantum parameter.'

This definition is identical to the one in Eq. (1), when we recall the expression $q \equiv \hbar\left(k_{\mathrm{L}}+k_{\mathrm{W}}\right)$ for the recoil. In Ref. [12], this expression was derived by rewriting the result in the Bambini-Renieri frame in terms of the laboratory frame.

We emphasize that the dependency of the quantum parameter in Eq. (45) on $\gamma^{2}$ does not mean that a low energy leads to a small $\alpha$. In Ref. [12], we have shown that $\alpha$ in fact is suppressed for increasing energies when we express the recoil $q \equiv \hbar\left(k_{\mathrm{L}}+k_{\mathrm{W}}\right)$ solely in terms of $\gamma$ and $k_{\mathrm{W}}$ and not in terms of the wave number $k_{\mathrm{L}}$ of the laser. Indeed, by inspecting Eqs. (27) and (28) and setting $\gamma_{\mathrm{BR}} \approx \gamma$ we obtain that $\alpha$ is proportional ${ }^{5}$ to $\gamma^{-2}$.

Energy spread The second requirement for a Quantum FEL derived in Ref. [12] concerns the relative energy spread $\Delta \gamma / \gamma$ of the electron beam. To obtain this condition in our present approach, we have to consider the initial momentum distribution $\left|\psi\left(p_{z}\right)\right|^{2}$ in the probability densities Eqs. (38) and (39).

A narrow momentum distribution of width $\Delta \beta$ localized at a resonance $\beta=\beta_{\mathrm{BR}} \pm \mathcal{Q} / 2$ prefers a single process, emission for $+\mathcal{Q} / 2$ or absorption for $-\mathcal{Q} / 2$. On the other hand, for a broad distribution that covers both resonances, both emission and absorption occur nearly with the same probability, and the net gain averages out.

The separation of the resonant velocities is given by the recoil parameter $\mathcal{Q}$ and we demand $\Delta \beta<\mathcal{Q}$ in order to obtain gain in the Quantum FEL. This condition can be cast into a more convenient form by approximating $\Delta \beta \approx \Delta \gamma / \gamma^{3}$ valid for relativistic energies, $\gamma \gg 1$. Thus, we arrive at the requirement

$\frac{\Delta \gamma}{\gamma}<\frac{\hbar\left(k_{\mathrm{L}}+k_{\mathrm{W}}\right)}{\gamma m c}$

where we have recalled the definition, Eq. (32), of the recoil parameter.

The right-hand side of Eq. (46) represents the ratio of the quantum mechanical recoil to the momentum of the electron in the laboratory frame. This ratio is of course very small which makes the condition on the energy spread of the electrons the most difficult hurdle to realize the Quantum FEL in an experiment.

We can also express the relation Eq. (46) in terms of the energy scaled $\gamma_{0}$ of the free electron, that is,

$\frac{\Delta \gamma_{0}}{\gamma_{0}}<\frac{4 \gamma}{1+a_{0}^{2}} \frac{\lambda_{\mathrm{C}}}{\lambda_{\mathrm{W}}}$

with the Compton wavelength $\lambda_{\mathrm{C}} \equiv h /\left(m_{0} c\right)$ of the electron. In the derivation of Eq. (47), we have used Eqs. (8) and (28), set $\gamma_{\mathrm{BR}} \cong \gamma$ and have employed the resonance condition, Eq. (27).

When we compare Eq. (47) with Eq. (2), derived in Ref. [12], we recognize that the formulas are similar with the difference that the effect of the effective momentum, visible in the factor $1+a_{0}^{2}$, was neglected in Ref. [12].

\footnotetext{
5 The proportionality of $\alpha$ to $\gamma^{-3}$ in Eq. (37) of Ref. [12] emerges when we take into account the dependencies of the coupling constant $g$. We omit this calculation here and refer the reader to Ref. [12].
} 
However, for reasonable parameters, $a_{0} \sim \mathcal{O}(1)$, both results differ only by a small amount.

\subsection{Higher-order processes}

So far, we have only considered single-photon processes which is in contrast to the classical FEL where many photons are relevant. This fact implies that many momentum levels are involved in the interaction.

Therefore, to prove that processes which include more than the two resonant momenta, $\mathcal{Q} / 2$ and $-\mathcal{Q} / 2$, are suppressed in the quantum regime, we have to take into account higher orders of the perturbative solution of Eq. (34). This calculation, which is analogous to the one presented in Ref. [12], is explicitly performed in Appendix C.

Indeed, we find that multi-photon processes are suppressed with powers of $\alpha \ll 1$, which is equivalent to a negligible population in higher momentum states. Thus, we really can identify the Quantum FEL as a two-level system for the momentum levels of the electron.

\section{Conclusion and outlook}

In conclusion, we have rederived the conditions for the Quantum FEL, Eqs. (45) and (47). Originally these constraints were introduced in Ref. [12]. We have now verified that both approaches, the relativistic one in the laboratory frame and the nonrelativistic in the Bambini-Renieri frame, yield equivalent results.

A relativistic effect which is not contained in our current model is the so-called slippage [38]. When no cavity is present, the laser field which propagates with the speed of light is slightly faster than the electrons, $\beta \lesssim 1$, and can slip over the electron bunch during the interaction. The inclusion of slippage and the development of a many-electron model, which is essential for the high-gain regime, represent necessary ingredients of a theory for a self-amplified spontaneous emission (SASE) FEL in the quantum regime. Both topics will be considered in future publications.

Acknowledgements We thank W. Becker, M. Bussmann, A. Debus, M. Knobl, K. Steiniger, S. Varró and M.S. Zubairy for many fruitful discussions. E.G. is grateful to the Center for Integrated Quantum Science and Technology (IQST) for a fellowship and the FriedrichAlexander-Universität Erlangen-Nürnberg for the Eugen Lommel Stipend. W.P.S. is thankful to Texas A\&M University for a Texas A\&M University Institute for Advanced Study (TIAS) Faculty Fellowship.

Open Access This article is distributed under the terms of the Creative Commons Attribution 4.0 International License (http://creativecommons.org/licenses/by/4.0/), which permits unrestricted use, distribution, and reproduction in any medium, provided you give appropriate credit to the original author(s) and the source, provide a link to the Creative Commons license, and indicate if changes were made.

\section{Appendix A: Furry picture for scalar quantum electrodynamics}

In this appendix, we derive, starting from basic principles, the model introduced in Sect. 2, namely the Furry or bound interaction picture [21] for a scalar field [19]. Here we first discuss the classical Klein-Gordon and Maxwell fields, as well as their interaction. Then we perform second quantization in the Schrödinger picture, before we finally investigate the transformation to the Furry picture. We conclude by presenting the Hamiltonian in the Furry picture.

\section{Lagrangian formulation}

We start from the classical Lagrangian density [39]

$\mathcal{L} \equiv \mathcal{L}_{\mathrm{K}-\mathrm{G}}+\mathcal{L}_{\mathrm{M}}+\mathcal{L}_{\text {Int }}$

for the Klein-Gordon field $\varphi=\varphi(x)$ interacting with an electromagnetic field described by the four-potential $A^{\mu}$.

The dynamics of the free Klein-Gordon field is determined by

$\mathcal{L}_{\mathrm{K}-\mathrm{G}} \equiv c\left[\hbar^{2} \partial_{\mu} \varphi^{*}(x) \partial^{\mu} \varphi(x)-m_{0}^{2} c^{2} \varphi^{*}(x) \varphi(x)\right]$

with the electron mass $m_{0}$, the speed of light $c$ and the reduced Planck constant $\hbar \equiv h / 2 \pi$.

The free Maxwell field evolves in time according to the Lagrangian density

$\mathcal{L}_{\mathrm{M}} \equiv-\frac{1}{4 \mu_{0}} F_{\mu \nu}(x) F^{\mu \nu}(x)$

where we have introduced the field tensor

$F^{\mu \nu}(x) \equiv \partial^{\mu} A^{\nu}(x)-\partial^{\nu} A^{\mu}(x)$

and the vacuum permeability $\mu_{0}$.

The interaction Lagrangian

$$
\begin{aligned}
\mathcal{L}_{\text {Int }} \equiv & -\mathrm{e} c A_{\mu}(x)\left[\varphi^{*}(x) i \hbar \partial^{\mu} \varphi(x)-\varphi(x) i \hbar \partial^{\mu} \varphi^{*}(x)\right] \\
& +\mathrm{e}^{2} c A^{2}(x) \varphi^{*}(x) \varphi(x)
\end{aligned}
$$

is chosen such that the Euler-Lagrange equation with respect to $\varphi^{*}(x)$, that is,

$\partial^{\mu} \frac{\partial \mathcal{L}}{\partial\left(\partial^{\mu} \varphi^{*}(x)\right)}-\frac{\partial \mathcal{L}}{\partial \varphi^{*}(x)}=0$,

leads to the Klein-Gordon equation

$\left[\left(i \hbar \partial^{\mu}-\mathrm{e} A^{\mu}(x)\right)^{2}-m_{0}^{2} c^{2}\right] \varphi(x)=0$

with minimal coupling $\hat{p}^{\mu} \rightarrow \hat{p}^{\mu}-\mathrm{e} A^{\mu}(x)$ between the operator $\hat{p}^{\mu} \equiv i \hbar \partial^{\mu}$ for the four-momentum and the potential $A^{\mu}(x)$. 
When we compute the corresponding Euler-Lagrange equations for $A^{\mu}$, we obtain the Maxwell equations

$\partial^{v} \partial_{v} A^{\mu}(x)=\mu_{0} \mathrm{e}^{\mu}(x)$,

where $A^{\mu}$ is driven by the four current

$$
\begin{aligned}
j^{\mu}(x) \equiv & c\left[\varphi^{*}(x) i \hbar \partial^{\mu} \varphi(x)-\varphi(x) i \hbar \partial^{\mu} \varphi^{*}(x)\right] \\
& +2 \mathrm{e} A^{\mu}(x) \varphi^{*}(x) \varphi(x),
\end{aligned}
$$

which represents a conserved quantity for Eq. (53), in the sense that $\partial_{\mu} j^{\mu}(x)=0$.

\section{Hamiltonian formulation}

In order to find a quantized theory for this interaction, we change to the Hamiltonian formalism of field theory. To simplify the calculations, we choose from the beginning the gauge

$\phi(x) \equiv \frac{1}{c} A^{(0)}(x)=0$

and

$\nabla \cdot \boldsymbol{A}(x)=0$

since we are only interested in the transverse degrees of freedom. In this way, we avoid that the momentum density

$\pi(x) \equiv \partial \mathcal{L} / \partial \dot{\varphi}(x)$

of the Klein-Gordon field is coupled to $\phi$. The fact that we recover the same results as if we would have retained $\phi$ and set it to zero at the end of the calculations [19] justifies this procedure.

With the help of Eq. (49), we obtain

$\pi(x)=\left(\hbar^{2} / c\right) \dot{\varphi}^{*}(x)$

and thus arrive at the Hamiltonian density

$\mathcal{H} \equiv \mathcal{H}_{\mathrm{K}-\mathrm{G}}+\mathcal{H}_{\mathrm{M}}+\mathcal{H}_{\text {Int }}$

with

$$
\begin{aligned}
\mathcal{H}_{\mathrm{K}-\mathrm{G}} \equiv & c\left[\frac{1}{\hbar^{2}} \pi^{*}(x) \pi(x)+\hbar^{2} \nabla \varphi^{*}(x) \cdot \nabla \varphi(x)\right. \\
& \left.+m_{0}^{2} c^{2} \varphi^{*}(x) \varphi(x)\right]
\end{aligned}
$$

for the free Klein-Gordon field,

$$
\begin{aligned}
\mathcal{H}_{\text {Int }} \equiv & \operatorname{e} c A^{\mu}(x)\left[\varphi^{*}(x) i \hbar \partial_{\mu} \varphi(x)-\left(i \hbar \partial_{\mu} \varphi^{*}(x)\right) \varphi(x)\right] \\
& -\mathrm{e}^{2} c A^{2}(x) \varphi^{*}(x) \varphi(x)
\end{aligned}
$$

for the interaction and $\mathcal{H}_{\mathrm{M}}$ as the Hamiltonian density [39] of the free Maxwell field.

\section{Quantization in Schrödinger picture}

Next we perform second quantization in the Schrödinger picture where the Klein-Gordon field operator $\hat{\varphi}_{S}(\boldsymbol{r})$ and its conjugate momentum $\hat{\pi}_{\mathrm{S}}(\boldsymbol{r})$ fulfill the commutation relations

$\left[\hat{\pi}_{\mathrm{S}}(\boldsymbol{r}), \hat{\varphi}_{\mathrm{S}}\left(\boldsymbol{r}^{\prime}\right)\right]=\left[\hat{\pi}_{\mathrm{S}}^{\dagger}(\boldsymbol{r}), \hat{\varphi}_{\mathrm{S}}^{\dagger}\left(\boldsymbol{r}^{\prime}\right)\right]=-i \hbar \delta\left(\boldsymbol{r}-\boldsymbol{r}^{\prime}\right)$

while all other possible combinations of commutators vanish.

We emphasize that the operators $\hat{\varphi}_{\mathrm{S}}(\boldsymbol{r})$ and $\hat{\pi}_{\mathrm{S}}(\boldsymbol{r})$ do not depend on time in contrast to their classical counterparts $\varphi(x) \equiv \varphi(c t, r)$ and $\pi(x) \equiv \pi(c t, r)$. In the Schrödinger picture, all dynamics is contained in the state vector $\left|\Psi_{\mathrm{S}}(t)\right\rangle$ for the electron and the electromagnetic field and follows from the Schrödinger equation

$i \hbar \frac{\mathrm{d}}{\mathrm{d} t}\left|\Psi_{\mathrm{S}}(t)\right\rangle=\hat{H}_{\mathrm{S}}\left|\Psi_{\mathrm{S}}(t)\right\rangle$.

Here we have replaced all fields and momenta by their operators in the Hamiltonian

$\hat{H}_{\mathrm{S}} \equiv \int \mathrm{d}^{3} r \hat{\mathcal{H}}_{\mathrm{S}}(\boldsymbol{r})$

The Furry picture is most convenient when the interaction with the electromagnetic field consists of two parts: one due to an external field which is treated as classical and fixed, which is its depletion is neglected, and one with a quantized field.

In our case, we identify these two components as the classical wiggler field $A_{\mathrm{W}}^{\mu}$ and the quantized laser field $\hat{A}_{\mathrm{L}}^{\mu}$, that is,

$\hat{A}^{\mu}(x)=\hat{A}_{\mathrm{L}}^{\mu}(\boldsymbol{r})+A_{\mathrm{W}}^{\mu}(x)$.

Here we have fixed the dynamics of the wiggler field in the time-dependent potential $A_{\mathrm{W}}(x) \equiv A_{\mathrm{W}}(c t, r)$, while the operator $\hat{A}_{\mathrm{L}}(\boldsymbol{r})$ is independent of time.

We can make a similar distinction of Eq. (66) for the Hamiltonian

$\hat{H}_{\mathrm{S}}(t) \equiv \hat{H}_{\mathrm{W}}(t)+\hat{H}_{\mathrm{L}} \equiv \int \mathrm{d}^{3} r\left[\hat{\mathcal{H}}_{\mathrm{W}}(t)+\hat{\mathcal{H}}_{\mathrm{L}}\right]$

with

$$
\begin{aligned}
\hat{\mathcal{H}}_{\mathrm{W}}(t) \equiv & c\left[\frac{1}{\hbar^{2}} \hat{\pi}_{\mathrm{S}}^{\dagger}(\boldsymbol{r}) \hat{\pi}_{\mathrm{S}}(\boldsymbol{r})+\hbar^{2} \nabla \hat{\varphi}_{\mathrm{S}}^{\dagger}(\boldsymbol{r}) \cdot \nabla \hat{\varphi}_{\mathrm{S}}(\boldsymbol{r})\right. \\
& +\left(m_{0}^{2} c^{2}-\mathrm{e}^{2} A_{\mathrm{W}}^{2}(x)\right) \hat{\varphi}_{\mathrm{S}}^{\dagger}(\boldsymbol{r}) \hat{\varphi}_{\mathrm{S}}(\boldsymbol{r})+\hat{\mathcal{H}}_{\mathrm{M}}(\boldsymbol{r}) \\
& \left.+\mathrm{e} A_{\mathrm{W}}^{\mu}(x)\left(\hat{\varphi}_{\mathrm{S}}^{\dagger}(\boldsymbol{r}) i \hbar \partial_{\mu} \hat{\varphi}_{\mathrm{S}}(\boldsymbol{r})-\left(i \hbar \partial_{\mu} \hat{\varphi}_{\mathrm{S}}^{\dagger}(\boldsymbol{r})\right) \hat{\varphi}_{\mathrm{S}}(\boldsymbol{r})\right)\right]
\end{aligned}
$$

and 


$$
\begin{aligned}
\hat{\mathcal{H}}_{\mathrm{L}}= & \mathrm{e} c \hat{A}_{\mathrm{L}}^{\mu}(\boldsymbol{r})\left[\hat{\varphi}_{\mathrm{S}}^{\dagger}(\boldsymbol{r}) i \hbar \partial_{\mu} \hat{\varphi}_{\mathrm{S}}(\boldsymbol{r})-\left(i \hbar \partial_{\mu} \hat{\varphi}_{\mathrm{S}}^{\dagger}(\boldsymbol{r})\right) \hat{\varphi}_{\mathrm{S}}(\boldsymbol{r})\right] \\
& -c\left[\mathrm{e}^{2} \hat{A}_{\mathrm{L}}^{2}(\boldsymbol{r})+2 e^{2} A_{\mathrm{W}}(x) \cdot \hat{A}_{\mathrm{L}}(\boldsymbol{x})\right] \hat{\varphi}_{\mathrm{S}}^{\dagger}(\boldsymbol{r}) \hat{\varphi}_{\mathrm{S}}(\boldsymbol{r}) .
\end{aligned}
$$

From these expressions, we observe two important aspects: (1) Although we use the Schrödinger picture, the Hamiltonian $\hat{H}_{\mathrm{W}}$ is explicitly time dependent. This feature originates from the fact that we have used for the wiggler a time-dependent classical field instead of a field operator in the Schrödinger picture. By prescribing the dynamics of the wiggler, we have already incorporated the free motion of this field mode.

(2) We realize that $\hat{H}_{\mathrm{L}}$ still contains a term linear in $A_{\mathrm{W}}^{\mu}$. This linear contribution arises as the cross-term to the square $A^{2}$ in Eq. (62) of the sum in Eq. (66). Later on, we shall recognize that this term is crucial for the FEL.

\section{Transformation to the Furry picture}

Similar to the transition into the familiar interaction picture, we obtain the Furry picture by transforming the original state $\left|\Psi_{\mathrm{S}}(t)\right\rangle$ by a unitary operator $V(t)$ such that

$\left|\Psi_{\mathrm{F}}(t)\right\rangle=V^{-1}(t)\left|\Psi_{\mathrm{S}}(t)\right\rangle$.

With the help of the Schrödinger equation, Eq. (64), the time derivative of the new state $\left|\Psi_{\mathrm{F}}(t)\right\rangle$ reads

$$
\begin{aligned}
i \hbar \frac{\mathrm{d}}{\mathrm{d} t}\left|\Psi_{\mathrm{F}}(t)\right\rangle= & V^{-1}(t)\left[-i \hbar \dot{V}(t) V^{-1}(t)\right. \\
& \left.+\left(\hat{H}_{\mathrm{W}}(t)+\hat{H}_{\mathrm{L}}\right)\right] V(t)\left|\Psi_{\mathrm{F}}(t)\right\rangle .
\end{aligned}
$$

If we demand the Schrödinger-like equation

$i \hbar \dot{V}(t)=\hat{H}_{\mathrm{W}}(t) V(t)$

for $V(t)$, we arrive at the time evolution

$i \hbar \frac{\mathrm{d}}{\mathrm{d} t}\left|\Psi_{\mathrm{F}}(t)\right\rangle=\hat{H}_{\mathrm{F}}(t)\left|\Psi_{\mathrm{F}}(t)\right\rangle$

with

$\hat{H}_{\mathrm{F}}(t) \equiv V^{-1}(t) \hat{H}_{\mathrm{L}} V(t)$

for the state $\left|\Psi_{\mathrm{F}}\right\rangle$ which is focused on the interaction with the laser field. Indeed, the Hamiltonian $\hat{H}_{\mathrm{F}}$ in this picture is a function of the transformed field operators

$\hat{\varphi}_{\mathrm{F}}(x) \equiv V^{-1}(t) \hat{\varphi}_{\mathrm{S}}(\boldsymbol{r}) V(t)$,

and

$\hat{\pi}_{\mathrm{F}}(x) \equiv V^{-1}(t) \hat{\pi}_{\mathrm{S}}(\boldsymbol{r}) V(t)$

with

$\hat{A}_{\mathrm{L}}^{\mu}(x) \equiv V^{-1}(t) \hat{A}_{\mathrm{L}}^{\mu}(\boldsymbol{r}) V(t)$.
When we examine Eq. (72), we notice the difficulties to specify the explicit form of the transformation matrix $V$ for the Furry picture, since we are dealing with the timedependent Hamiltonian $\hat{H}_{\mathrm{W}}$. Indeed, we find the timeordered exponential [36]

$V(t)=\mathcal{T} \exp \left[-\frac{i}{\hbar} \int \mathrm{d} t \hat{H}_{\mathrm{W}}(t)\right]$

for $V(t)$, in contrast to the simple exponential in the ordinary Dirac picture.

Only the operator of the electromagnetic field can be written in the same form as in the familiar interaction picture, because the Hamiltonian of the Maxwell field commutes with the other contributions in $\hat{H}_{\mathrm{W}}$.

Nevertheless, we can formulate the equation of motion

$\frac{\partial}{\partial t} \hat{\varphi}_{\mathrm{F}}(x)=\frac{i}{\hbar} V^{-1}(t)\left[\hat{H}_{\mathrm{W}}(t), \hat{\varphi}_{\mathrm{S}}(\boldsymbol{r})\right] V(t)$

for the field operator of the Klein-Gordon field where we have used Eq. (72) and the fact that $\hat{\varphi}_{S}(\boldsymbol{r})$ is independent of time in the Schrödinger picture.

The evaluation of the commutator in Eq. (79), with the help of Eqs. (63) and (68), yields

$\frac{\partial}{\partial t} \hat{\varphi}_{\mathrm{F}}(x)=\frac{c}{\hbar^{2}} \hat{\pi}_{\mathrm{F}}^{\dagger}(x)$.

In a similar way, we compute

$$
\begin{aligned}
\frac{\partial^{2}}{\partial t^{2}} \hat{\varphi}_{\mathrm{F}}(x) & =\frac{c}{\hbar^{2}} \frac{\partial}{\partial t} \hat{\pi}_{\mathrm{F}}^{\dagger}(x) \\
& =\frac{c}{\hbar^{2}} \frac{i}{\hbar} V^{-1}(t)\left[\hat{H}_{\mathrm{W}}(t), \hat{\pi}_{\mathrm{S}}^{\dagger}(\boldsymbol{r})\right] V(t),
\end{aligned}
$$

and observe that the field operator in the Furry picture fulfills the Klein-Gordon equation in the external wiggler field

$\left[\left(i \hbar \partial^{\mu}-\mathrm{e} A_{\mathrm{W}}^{\mu}(x)\right)^{2}-m_{0}^{2} c^{2}\right] \hat{\varphi}_{\mathrm{F}}(x)=0$.

Hence, we can expand the field operator in terms of solutions of this equation of motion. This procedure is analogous to the ordinary interaction picture, where the field operator is expanded in solutions of the free Klein-Gordon equation. The task is, of course, to find a solution of this external field problem, Eq. (82), which in case of a plane wave field is given by the Volkov solution [22], as discussed in Appendix B.

\section{Hamiltonian in the Furry picture}

We conclude by noting that the interaction Hamiltonian defined by Eq. (74) can be written as

$\hat{H}_{\mathrm{F}}=\mathrm{e} \int \mathrm{d}^{3} r \hat{j}_{\mathrm{F}}(x) \cdot \hat{A}_{\mathrm{L}}(x)$ 
where the electron current $\hat{j}$ couples to the laser field $\hat{A}_{\mathrm{L}}$. Here we have neglected the term proportional to $\hat{A}_{L}^{2}(x)$ and have recalled from Eq. (55) that the current

$$
\begin{aligned}
\hat{j}_{\mathrm{F}}^{\mu}(x) \equiv & c\left[\hat{\varphi}_{\mathrm{F}}^{\dagger}(x) i \hbar \partial^{\mu} \hat{\varphi}_{\mathrm{F}}(x)-\left(i \hbar \partial^{\mu} \hat{\varphi}_{\mathrm{F}}^{\dagger}(x)\right) \hat{\varphi}_{\mathrm{F}}(x)\right] \\
& +2 \mathrm{e} A_{\mathrm{W}}^{\mu}(x) \hat{\varphi}_{\mathrm{F}}^{\dagger}(x) \hat{\varphi}_{\mathrm{F}}(x)
\end{aligned}
$$

is conserved.

For the sake of clarity, we omit in the main body of this article the subscript F indicating the Furry picture. Since, apart from this appendix, no quantities outside the framework of the Furry picture are considered, this procedure seems justified.

\section{Appendix B: Volkov solution for Klein-Gordon equation}

The term 'Volkov solution' usually refers to a solution of the Dirac equation coupled to an external electromagnetic field in the form of a plane wave and was derived in 1935 by D. M. Volkov [22]. However, in the present context 'Volkov solution' refers to a solution of the corresponding problem of the Klein-Gordon equation. In this appendix, we rederive [19] the Volkov solution in the case of an optical undulator described by the potential

$A_{\mathrm{W}}^{\mu}=\tilde{\mathcal{A}}_{\mathrm{W}}\left(\epsilon^{\mu} \mathrm{e}^{-i k_{\mathrm{W}} \cdot x}+\right.$ c.c. $)$

given by Eq. (4).

In order to solve the equation

$\left[\left(i \hbar \partial^{\mu}-\mathrm{e} A_{\mathrm{W}}^{\mu}(x)\right)^{2}-m_{0}^{2} c^{2}\right] \varphi(x)=0$

we note that $A_{\mathrm{W}}^{\mu}$ depends only on the phase $\xi \equiv k_{\mathrm{W}} \cdot x$ and assume that the free field solution is only modified by a function $f=f(\xi)$. This assumption gives rise to the ansatz

$\varphi(x)=\mathcal{N} f(\xi) \mathrm{e}^{-i p_{\mathrm{f}} \cdot x / \hbar}$.

Here $p_{\mathrm{f}}^{\mu}$ describes the free four-momentum of the electron and fulfills the relation $p_{\mathrm{f}} \cdot p_{\mathrm{f}}=m_{0}^{2} c^{2}$, while $\mathcal{N}$ is a normalization constant.

In addition, we demand for a one-dimensional theory that before the electron enters, the wiggler its momentum is parallel to the propagation direction of the field, corresponding to the relation

$p_{\mathrm{f}} \cdot A_{\mathrm{W}}^{\mu}(x)=p_{\mathrm{f}} \cdot \hat{A}_{\mathrm{L}}^{\mu}(x)=0$.

Since the potentials are transverse, all scalar products between the four-wave vectors and the four-potentials vanish, and in particular, we have
$k_{\mathrm{W}} \cdot A_{\mathrm{W}}=0$

for the wiggler.

Under these assumptions, we insert the ansatz Eq. (87) into Eq. (86) and obtain the first-order differential equation

$f^{\prime}(\xi)=-\frac{i}{\hbar} \frac{\mathrm{e}^{2} \tilde{\mathcal{A}}_{\mathrm{W}}^{2}}{p_{\mathrm{f}} \cdot k_{\mathrm{W}}} f(\xi)$

for $f$ with $f^{\prime}(\xi) \equiv \mathrm{d} f(\xi) / \mathrm{d} \xi$. Here we have made use of the identity $A_{\mathrm{W}}^{2}(x)=-2 \tilde{\mathcal{A}}_{\mathrm{W}}^{2}$ valid for circular polarization.

Crucial for the change from a second-order differential equation, Eq. (86), to a first-order one, Eq. (90), is the fact that the only term with a second derivative, that is, the contribution $-\hbar^{2} k_{\mathrm{W}} \cdot k_{\mathrm{W}} f^{\prime \prime}(\xi)$ vanishes due to the dispersion relation $k_{\mathrm{W}} \cdot k_{\mathrm{W}}=0$ for the wiggler field.

Although the dispersion relation for the four-wave vector is correct only in the case of a laser wiggler the second derivative can still be neglected $[19,20]$ in the magnetostatic case. Indeed, the corrections due to the second derivative scale with $a_{0}^{2} / \gamma_{0}^{2}$, which is negligible for all reasonable values of the field strength of a wiggler.

The solution of the differential equation, Eq. (90), reads

$f(\xi)=\exp \left[-\frac{i}{\hbar} \frac{\mathrm{e}^{2} \tilde{\mathcal{A}}_{\mathrm{W}}^{2}}{p_{\mathrm{f}} \cdot k_{\mathrm{W}}} \xi\right]$

where we have paid attention to the constraint that $f$ should reduce to $f(\xi)=1$ for a vanishing wiggler field, that is, for $A_{\mathrm{W}}^{\mu}(x)=0$.

When we combine all terms we obtain the Volkov solution [19]

$\varphi_{p}(x)=\frac{1}{\sqrt{2 p_{0} V}} \mathrm{e}^{-i p \cdot x / \hbar}$,

where we have defined the effective momentum

$p^{\mu}=p_{\mathrm{f}}^{\mu}+\frac{\mathrm{e}^{2} \tilde{\mathcal{A}}_{\mathrm{W}}^{2}}{p_{\mathrm{f}} \cdot k_{\mathrm{W}}} k_{\mathrm{W}}^{\mu}$

of the electron in the wiggler field which fulfills the modified energy-momentum relation

$p \cdot p=p_{0}^{2}-\boldsymbol{p}^{2}=m_{0}^{2} c^{2}+2 \mathrm{e}^{2} \tilde{\mathcal{A}}^{2} \equiv m^{2} c^{2}$

with the effective mass $m$.

The normalization constant $\mathcal{N}$ follows from the condition that the integral of the density, i.e., zeroth component of the four current, Eq. (55), divided by $c$, over the quantization volume $V$ yields unity, that is,

$\int_{V} \mathrm{~d}^{3} r\left[\varphi_{p}^{*}(x) \frac{i \hbar}{c} \frac{\partial}{\partial t} \varphi_{p}(x)-\varphi_{p}(x) \frac{i \hbar}{c} \frac{\partial}{\partial t} \varphi_{p}^{*}(x)\right]=1$ 
. In conclusion, we observe that, similar to the free field case, the solution of Eq. (86) is a plane wave where the free momentum $p_{\mathrm{f}}$ is replaced by the effective momentum $p$ which takes the effect of the wiggler field into account. Due to the linearity of the problem any other solution of Eq. (86) can be expanded in plane waves of this kind.

\section{Appendix C: Two-photon processes}

In this appendix, we show that higher-order photon processes are suppressed. For this purpose, we first analyze the influence of two-photon transitions within our perturbative approach and demonstrate that they scale with $\alpha^{2}$. We conclude by briefly analyzing the general case.

The second-order terms in the expression Eq. (34) for the time evolution operator lead to the evolved state

$\left|\Psi^{(2)}(t)\right\rangle \equiv \int_{0}^{t} \mathrm{~d} t_{2} \int_{0}^{t_{2}} \mathrm{~d} t_{1} \hat{H}\left(t_{2}\right) \hat{H}\left(t_{1}\right)\left|n, p_{z}\right\rangle$.

for the initial state $\left|n, p_{z}\right\rangle$. Apart from the contributions with $\hat{\mathcal{H}}_{\text {em }}\left(t_{2}\right) \hat{\mathcal{H}}_{\text {em }}\left(t_{1}\right)$ representing two-photon emission, and with $\hat{\mathcal{H}}_{\text {abs }}\left(t_{2}\right) \hat{\mathcal{H}}_{\text {abs }}\left(t_{1}\right)$ representing two-photon absorption, there also emerge cross-terms with $\hat{\mathcal{H}}_{\text {abs }}\left(t_{2}\right) \hat{\mathcal{H}}_{\mathrm{em}}\left(t_{1}\right)$ and $\hat{\mathcal{H}}_{\text {em }}\left(t_{2}\right) \hat{\mathcal{H}}_{\text {abs }}\left(t_{1}\right)$ describing processes where the number of photons remains unchanged. We omit the latter ones and discuss only the two-photon transitions.

From a calculation analogous to the single-photon case we obtain the probability density

$\left|c_{2}\right|^{2}=\left(\frac{g t}{\gamma}\right)^{4}(n+1)(n+2) \mathcal{E}^{(+2)}\left|\psi\left(p_{z}\right)\right|^{2}$

for two-photon emission, and the probability density

$\left|c_{-2}\right|^{2}=\left(\frac{g t}{\gamma}\right)^{4} n(n-1) \mathcal{E}^{(-2)}\left|\psi\left(p_{z}\right)\right|^{2}$

for two-photon absorption. Both expressions are weighted with the initial momentum distribution $\left|\psi\left(p_{z}\right)\right|^{2}$.

Moreover, we have defined the selectivity functions

$$
\begin{aligned}
\mathcal{E}^{( \pm 2) \equiv} & \frac{1}{\left(2 \phi_{ \pm 1}\right)^{2}}\left[\operatorname{sinc}^{2}\left(2 \phi_{ \pm 2}\right)+\operatorname{sinc}^{2} \phi_{ \pm 3}\right. \\
& \left.-2 \cos \phi_{ \pm 1} \operatorname{sinc}\left(2 \phi_{ \pm 2}\right) \operatorname{sinc} \phi_{ \pm 3}\right]
\end{aligned}
$$

for these processes with the phases

$\phi_{ \pm j} \equiv \frac{\left(k_{\mathrm{L}}+k_{\mathrm{W}}\right) c t}{2}\left(\beta-\beta_{\mathrm{BR}} \mp j \frac{\mathcal{Q}}{2}\right)$.

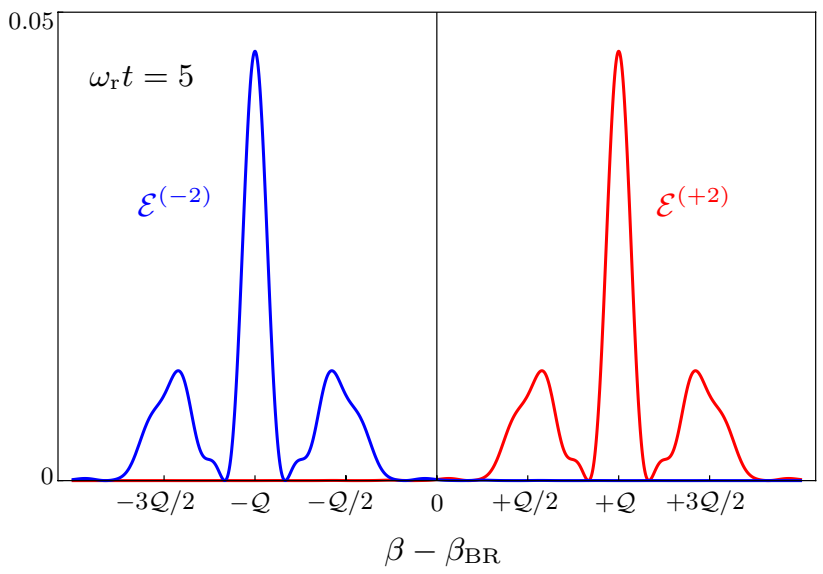

Fig. 2 Selectivity functions $\mathcal{E}^{(+2)}$ (red curve) or $\mathcal{E}^{(-2)}$ (blue curve) for two-photon emission or absorption, defined by Eq. (99), in the quantum regime for $\omega_{\mathrm{r}} t=5$, as functions of the scaled velocity $\beta$ of the electron. Although minor maxima exist, the dominant ones are located at $\beta=\beta_{\mathrm{BR}} \pm \mathcal{Q}$ as predicted by energy-momentum conservation

As shown in Fig. 2, the dominant maxima of $\mathcal{E}^{( \pm 2)}$ are located at $\beta=\beta_{\mathrm{BR}} \pm \mathcal{Q}$ as expected from energy-momentum conservation, Eq. (31). However, there are also less pronounced maxima separated from these major ones by $\mathcal{Q} / 2$.

The probability for two-photon emission, Eq. (97), is maximized for $\beta-\beta_{\mathrm{BR}}=\mathcal{Q}$ where we can estimate

$\mathcal{E}^{(+2)} \cong \frac{1}{\left(2 \omega_{\mathrm{r}} t\right)^{2}}$.

Thus, the probability for this process scales as

$\left|c_{2}\right|^{2} \sim\left(\frac{g t}{\gamma}\right)^{2}(n+2) \frac{\alpha^{2}}{4}$

where we have used the definitions Eqs. (44) and Eq. (45) of the recoil frequency and the quantum parameter $\alpha$, respectively.

On the other hand, the maximum probability for singlephoton emission, Eq. (38), scales with

$\left|c_{1}\right|^{2} \sim\left(\frac{g t}{\gamma}\right)^{2}(n+1)$

and we arrive at the ratio

$\frac{\left|c_{2}\right|^{2}}{\left|c_{1}\right|^{2}} \sim \frac{\alpha^{2}}{4}$

which we have also obtained in Ref. [12] in an analogous way. 
This relation demonstrates that in a Quantum FEL defined by $\alpha \ll 1$, two-photon emission is strongly suppressed in comparison with the single-photon transition. Since processes involving more than two photons occur only at even higher orders of perturbation theory, we argue that these processes are suppressed with corresponding powers of $\alpha$.

\section{References}

1. W.B. Colson, Phys. Lett. A 64, 190 (1977)

2. F.A. Hopf, P. Meystre, M.O. Scully, W.H. Louisell, Opt. Commun. 18, 413 (1976)

3. Z. Huang, K.-J. Kim, Phys. Rev. Spec. Top. Accel. Beams 10, 034801 (2007)

4. R. Bonifacio, N. Piovella, G.R.M. Robb, Fortschr. Phys. 57, 1041 (2009)

5. C.B. Schroeder, C. Pellegrini, P. Chen, Phys. Rev. E 64, 056502 (2001)

6. R. Bonifacio, N. Piovella, M.M. Cola, L. Volpe, A. Schiavi, G.R.M. Robb, Nucl Instrum Methods Phys. Res. Sect. A Accel. Spectrom. Detect. Assoc. Equip. 593, 69 (2008)

7. H.K. Avetissian, G.F. Mkrtchian, Phys. Rev. E 65, 046505 (2002)

8. H.K. Avetissian, G.F. Mkrtchian, Phys. Rev. Spec. Top. Accel. Beams 10, 030703 (2007)

9. A. Serbeto, L.F. Monteiro, K.H. Tsui, J.T. Mendonça, Plasma Phys. Control. Fusion 51, 124024 (2009)

10. B. Eliasson, P.K. Shukla, Plasma Phys. Control. Fusion 54, 124011 (2012)

11. B. Eliasson, P.K. Shukla, Phys. Rev. E 85, 065401 (2012)

12. P. Kling, E. Giese, R. Endrich, P. Preiss, R. Sauerbrey, W.P. Schleich, New J. Phys. 17, 123019 (2015)

13. A. Bambini, A. Renieri, Lett. Nuovo Cimento 21, 399 (1978)

14. A. Bambini, A. Renieri, S. Stenholm, Phys. Rev. A 19, 2013 (1979)

15. P. Preiss, R. Sauerbrey, M. S. Zubairy, R. Endrich, E. Giese, P. Kling, M. Knobl, W. P. Schleich, in Proceedings of the 34th Free-Electron Laser Conference, Nara, Japan, (2012), pp. 93-96

16. R. Endrich, E. Giese, P. Kling, R. Sauerbrey, W. P. Schleich, in Proceedings of the 36th Free-Electron Laser Conference, Basel, Switzerland, (2014), pp. 353-357
17. P. Kling, R. Endrich, E. Giese, R. Sauerbrey, W. P. Schleich, in Proceedings of the 36th Free-Electron Laser Conference, Basel Switzerland, (2014), pp. 348-352

18. E.T. Jaynes, F.W. Cummings, Proc. IEEE 51, 89 (1963)

19. W. Becker, J. McIver, Z. Phys. D Atoms Mol. Clust. 7, 353 (1988)

20. J. Gea-Banacloche, Phys. Rev. A 31, 1607 (1985)

21. W. H. Furry, Phys. Rev. 81, 115 (1951)

22. D.M. Wolkow, Z. Phys. 94, 250 (1935)

23. W. Becker, J.K. McIver, J. Phys. Colloq. 44, C1 (1983)

24. J.K. McIver, M.V. Fedorov, J. Exp. Theor. Phys. 49, 1012 (1979)

25. W. Becker, J. K. McIver, Phys. Rev. A 27, 1030 (1983)

26. M.V. Fedorov, Atomic and Free Electrons in a Strong Light Field (World Scientific, Singapore, 1997)

27. R.R. Schlicher, M.O. Scully, H. Walther, Vorschlag für einen kompakten Freie-Elektronen Laser mit einem elektromagnetischen Undulator für den Infrarot- und weichen Röntgenbereich, Tech. rep, Max-Planck Institut für Quantenoptik (1987)

28. J. Gea-Banacloche, G.T. Moore, R.R. Schlicher, M.O. Scully, H. Walther, IEEE J. Quantum Electron. 23, 1558 (1987)

29. P. Sprangle, B. Hafizi, J. Peñano, Phys. Rev. Spec. Top. Accel. Beams 12, 050702 (2009)

30. K. Steiniger, M. Bussmann, R. Pausch, T. Cowan, A. Irman, A. Jochmann, R. Sauerbrey, U. Schramm, A. Debus, J. Phys. B Atomic Mol. Opt. Phys. 47, 234011 (2014)

31. R. Bonifacio, N. Piovella, G.R.M. Robb, Nucl Instrum Methods Phys. Res. Sect. A Accel. Spectrom. Detect. Assoc. Equip. 543, 645 (2005)

32. W. Becker, H. Mitter, Z. Phys. B Condens. Matter 35, 399 (1979)

33. S. Varró, Intensity Effects and Absolute Phase Effects in Nonlinear Laser-Matter Interactions (InTech, Rijeka, 2010)

34. W. Becker, Opt. Commun. 33, 69 (1980)

35. P. Schmüser, M. Dohlus, J. Rossbach, Ultraviolet and Soft X-Ray Free-Electron Lasers (Springer, Heidelberg, 2008)

36. W.P. Schleich, Quantum Optics in Phase Space (Wiley, Weinheim, 2001)

37. F. Ciocci, G. Dattoli, A. Renieri, A. Torre, Phys. Rep. 141, 1 (1986)

38. R. Bonifacio, L. De Salvo, P. Pierini, N. Piovella, C. Pellegrini, Phys. Rev. Lett. 73, 70 (1994)

39. J. D. Bjorken, S. D. Drell, Relativistische Quantenfeldtheorie (Bibliographisches Institut, Mannheim, 1967) 